\title{
Possible role of the RhoC/ROCK pathway in progression of clear cell renal cell carcinoma
}

Hideyuki Abe*, Takao Kamai*, Toshihiko Tsuji, Fumihiko Nakamura, Tomoko Mashidori, Tomoya Mizuno, Miho Tanaka, Katsuhisa Tatsumiya, Nobutaka Furuya, Akinori Masuda, Tomonori Yamanishi and Ken-Ichiro YOSHIDA

Department of Urology, Dokkyo Medical University, Tochigi, Japan.

(Received 1 February 2008; and accepted 18 April 2008)

\begin{abstract}
To clarify the role of the Rho small GTP-binding protein (Rho) and its major downstream target, ROCK (Rho-associated serine-threonine protein kinase), in progression of renal cell carcinoma (RCC), we examined mRNA expression for Rho and ROCK genes in surgical specimen of RCC tissues from 78 Japanese patients and in the corresponding non-tumor tissues originating from the same patient using a real-time reverse transcription polymerase chain reaction (RT-PCR). Expression of mRNA for RhoA did not differ between tumor and non-tumor tissues. RhoB mRNA expression was higher in the tumor $(P<0.05)$, but expression was not associated with tumor grade, stage, or prognosis. However, degree of RhoC and ROCK mRNA expression was related to tumor grade $(P<0.05)$ and stage $(P<0.0001)$. A positive relationship was seen between expression of mRNA for RhoC and that for ROCK in tumor tissues $(P<0.0001)$. Kaplan-Meier plots showed high RhoC and ROCK mRNA expression to be negatively associated with overall survival $(P<0.0001)$. Multivariate analysis showed mRNA expression of RhoC and ROCK to be independent poor prognostic factors concerning overall survival. Our findings implicate the RhoC/ROCK pathway in carcinogenesis and progression of $\mathrm{RCC}$, indicating that RhoC/ROCK may be a useful prognostic marker and a possible molecular target for treatment of the disease.
\end{abstract}

Annual estimates of new diagnoses of renal cell carcinoma (RCC) are increasing steadily (17). RCC is resistant to chemotherapy and radiotherapy $(17,28$, 29 ), while immunotherapy with interferon and/or interleukin (IL)-2 achieves responses in $10 \%$ to $20 \%$ of advanced RCC $(17,18)$. Although surgical resection of the primary tumor for patients remains the mainstay of therapy, RCC is characterized by a high proportion of cases with metastases present at diagnosis or appearing as a relapse following nephrectomy. Patients with distant metastases have a poor prognosis, with a 5-year survival rate of less than

Address correspondence to: Takao Kamai, M. D. Ph. D. Department of Urology, Dokkyo Medical University, 880 Kitakobayashi, Mibu, Tochigi, 321-0293, Japan

Tel: +81-282-87-2162, Fax: +81-282-86-7533

E-mail: kamait@dokkyomed.ac.jp

*H. A. and T. K. were equally contributed to this work.
$10 \%$ in stage IV disease (17). Although inhibition of metastases logically would be a promising treatment strategy in advanced RCC, much of the molecular mechanism of progression and metastasis in RCC is yet to be eluidated.

Cell migration is central to metastasis of malignant tumors (20). The Rho small GTP-binding protein (Rho) regulates formation of stress fibers, focal adhesions, and cell migration through reorganization of the actin cytoskeleton $(7,25)$. Overexpression of Rho in human cancer tissues in comparison to nontumor tissues has been reported, with higher expression of Rho correlating with higher stage $(6,13$, 24). Several lines of evidence directly link Rho to acquisition of a migratory, invasive, and metastatic phenotype $(2,3)$. Furthermore, Rho-kinase (ROCK), one of the major downstream effectors of Rho, induces stress-fiber formation and assembly for focal contact by regulating contractility of the actin-myo- 
sin system $(9,16)$. ROCK has been reported to be involved in progression of the human tumors $(8,10$, 12), while a ROCK inhibitor suppressed tumor growth and metastasis $(8,10)$. These observations suggest that the Rho/ROCK pathway may be associated with progression and metastasis in human tumors. However, we have known of no available data regarding this pathway in RCC. To address the question of whether the Rho/ROCK pathway is involved in renal carcinogenesis and progression of RCC, we compared degree of mRNA expression for Rho and ROCK genes in RCC tissue with expression in corresponding non-tumor parts of the same patient's nephretomy specimen using a real-time reverse transcription polymerase chain reaction (RTPCR). We also examined the relationship between intensity of expression in tumors and grade, stage, and prognosis. Such information may be useful for predicting the biological behavior of RCC and formulating treatment strategy in individual cases. We then considered the clinical implications of our molecular findings.

\section{MATERIALS AND METHODS}

Patients and tissue preparation. Specimens from surgery for newly diagnosed clear cell RCC between 1995 and 2000 from 78 consecutive Japanese patients were studied (46 men and 32 women; mean age, 65.8 years; range, 33-82). All patients underwent imaging studies (CT and/or MRI) prior to surgery to obtain information necessary for staging. Postoperative follow-up ranged from 3 to 78 months (median follow-up duration, 31 months). In all cases three sites from the tumor and three non-tumor samples from the resected kidney were studied (14). Resected tissues were embedded in optimal cutting temperature (OCT) tissue compound (Miles, Elkhart, IN) and stored at $-80^{\circ} \mathrm{C}$ as described previously (13, 24). Clinical stage was determined according to the criteria of the TNM system (23).

Real-time RT-PCR assay. Methods of purification of total RNA and cDNA synthesis were described previously (14). Expression profiles of the Rho and ROCK genes were analyzed with an ABI PRISM 7700 sequence detection system (Applied Biosystems, Foster City, CA) using the SYBR Green method. Oligonucleotide primer pairs for Rho and ROCK genes and $\beta 2$-microglobulin amplification, as well as conditions of PCR, were chosen as described previously $(12,13,19,24)$. A real-time RT-PCR assay was performed on $25 \mu \mathrm{L}$ of reaction mixture con- taining $20 \mathrm{ng}$ of sample cDNA, $100 \mathrm{nM}$ sense primer, $100 \mathrm{nM}$ antisense-primer, and $12.5 \mu \mathrm{L}$ of SYBR Green PCR Master Mix (Applied Biosystems). PCR was carried out for 50 cycles of $95^{\circ} \mathrm{C}$ for $15 \mathrm{sec}$ and $60^{\circ} \mathrm{C}$ for $1 \mathrm{~min}$. To normalize the amplified products in each sample, we used $\beta 2$-microglobulin as a quantitative internal control $(13,14)$. A standard curve for expression of each mRNA was generated using fivefold dilutions of a control RNA sample $(25 \mathrm{x}, 5 \mathrm{x}, 1 \mathrm{x}, 0.2 \mathrm{x}$, and $0.04 \mathrm{x})$. Expression of mRNA for Rho and ROCK genes was expressed as a ratio to that of $\beta 2$-microglobulin, and this relative expression was calculated $(13,14)$. Mean values from real-time RT-PCR data for the three samples of resected tissues were used for analysis according to a method described previously $(13,14)$.

Statistical analysis. Results of RT-PCR were analyzed statistically using the Mann-Whitney $U$ test as described previously $(13,14,24)$. Prognostic variables were analyzed for overall survival by the Cox proportional hazards model. The Kaplan-Meier method was used to estimate survival as a function of time, and survival differences were analyzed by the log-rank test. $P$ values less than 0.05 were considered to indicate statistical significance. Data were analyzed with commercial software.

\section{RESULTS}

mRNAs of RhoA, RhoB, and RhoC were detected in both tumor and non-tumor tissues. ROCK mRNAs could be detected clearly in tumor tissues, but were only weakly expressed in non-tumor tissues. Relative mRNA expression for RhoA did not show a significant difference between tumor and non-tumor tissues (mean \pm S.D. $=1.35 \pm 0.55$ vs. 1.25 $\pm 0.35, P=0.1716$ ) (Fig. 1A). Amounts of mRNA expressing RhoB, RhoC, and ROCK were significantly higher in tumor tissues than in non-tumor parts of specimens (RhoB, $1.48 \pm 0.87$ vs. $1.18 \pm$ $0.57, P=0.0483$, Fig. $1 \mathrm{~B}$; RhoC, $3.31 \pm 2.15$ vs 1.19 $\pm 0.66, P<0.0001$, Fig. $1 C$; ROCK, $1.83 \pm 0.95$ vs $1.13 \pm 0.28, P<0.0001$, Fig. $2 \mathrm{D})$.

High mRNA expression for RhoC and ROCK was associated with a poor histologic differentiation grade $(P<0.0001$; Fig. $2 \mathrm{~B}, \mathrm{C})$ and higher clinical stage $(P<0.0001$; Fig. 2E, F). In contrast, degree of mRNA expression for RhoB did not correlate with grade or stage (Fig. 2A, D). Expression of mRNA encoding ROCK in tumors correlated with expression of RhoC (correlation coefficient $r=0.581$, $P<0.0001$, Fig. 3), but this was not true for RhoB 

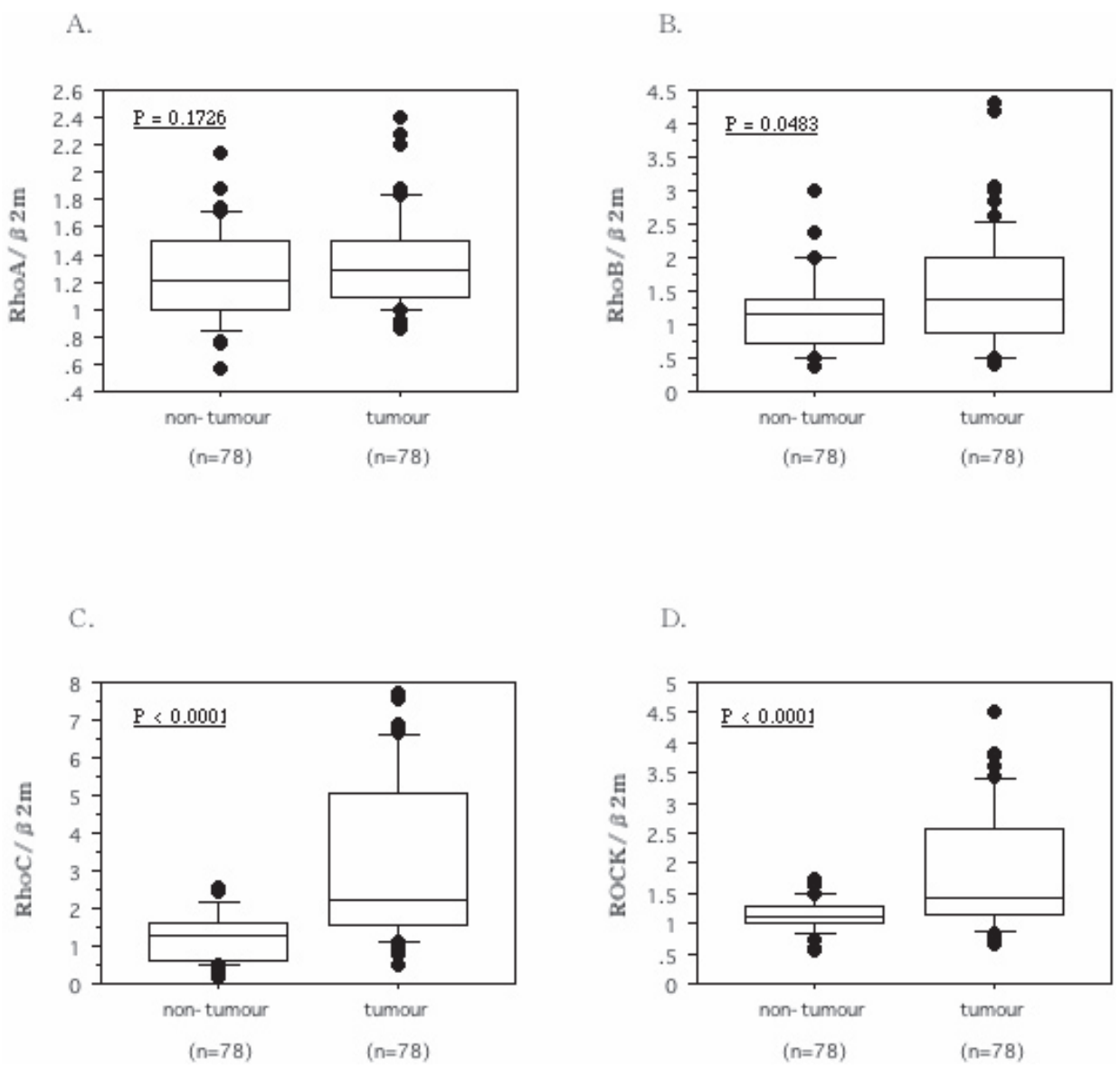

Fig. 1 The relative expression levels for mRNA of RhoA, RhoB, RhoC, and ROCK to those of $\beta 2$-microglobulin (RhoA/ $\beta 2 \mathrm{~m}, \mathrm{RhoB} / \beta 2 \mathrm{~m}$, RhoC/ $/ 32 \mathrm{~m}$, and $\mathrm{ROCK} / \beta 2 \mathrm{~m})$ in non-tumor and tumor. The median value was seen in the box-plots.

(data not shown).

We analyzed the cancer specific overall survival curve for RCC patients as follows. Mean values of relative mRNA expression for RhoB, RhoC, and ROCK in tumor tissues were $1.48( \pm 0.87), 3.31$ $( \pm 2.15)$, and $1.83( \pm 0.95)$, respectively. Cases were divided into two groups with expression above or below these means, high expression or low expression, according to methods described previously (24). Kaplan-Meier plots of survival rate in patients with low vs. high RhoC and ROCK mRNA expression showed that higher expression of RhoC and ROCK was associated with poorer overall survival $(P<$ 0.0001 ; Fig. 4B, C), while RhoB expression was not (Fig. 4A). Although a univariate analysis using the Cox proportional hazards model identified grade, stage, RhoC, and ROCK independent prognostic factors for overall survival, stage, RhoC, and ROCK remained significant in a multivariate analysis (Table 1).

\section{DISCUSSION}

As RCC metastasizes early by a hematogenous route, prognosis is generally unfavorable (17). Rho and its downstream effector, ROCK, are involved in regulation of a variety of cellular processes such as cytoskeletal organization, cell cycle progression, malignant transformation, and metastatic properties (3). To take into account the possibility of inter-individual variation in expression of mRNA for Rho and ROCK genes, tumor tissues in RCC and corresponding non-tumor tissues obtained from the same patient were compared. We found that mRNA expression for RhoC and ROCK were significantly elevated in tumors compared with non-tumor tissues. A positive correlation was present between RhoC and ROCK of mRNA expression in tumors. High RhoC and ROCK mRNA expression in tumor tissues were associated with higher tumor grade and stage. Furthermore, higher mRNA expression for RhoC and ROCK was associated with shortened 
A. RhoB mRNA levels in Grade

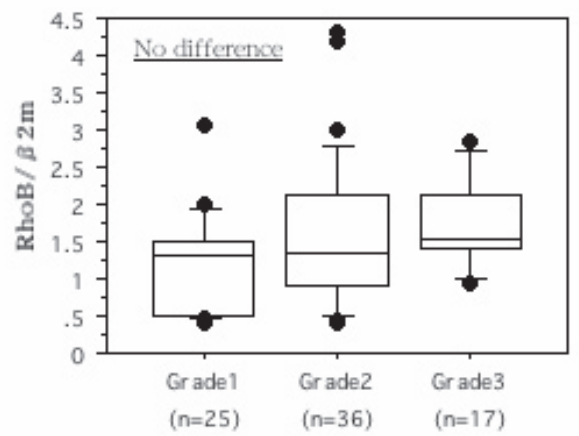

B. RhoC mRNA levels in Grade

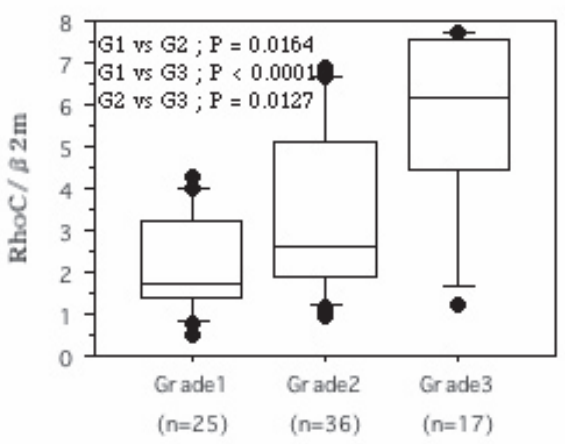

C. ROCK mRNA levels in Grade

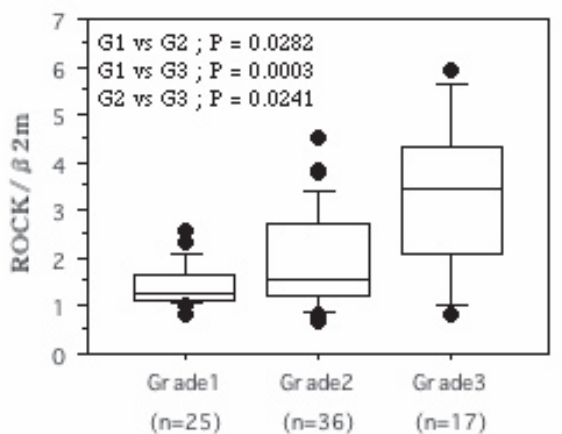

D. RhoB mRNA levels in Stage

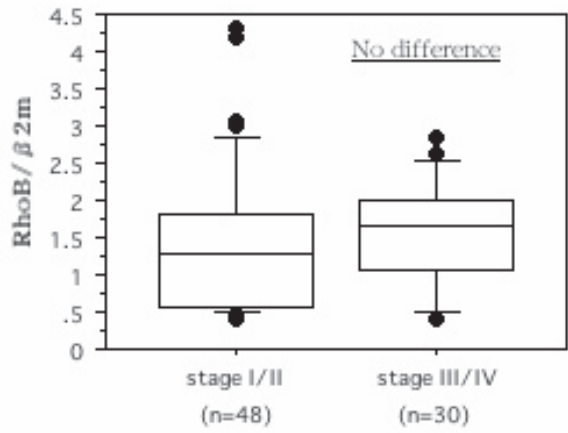

E. RhoC mRNA levels in Stage

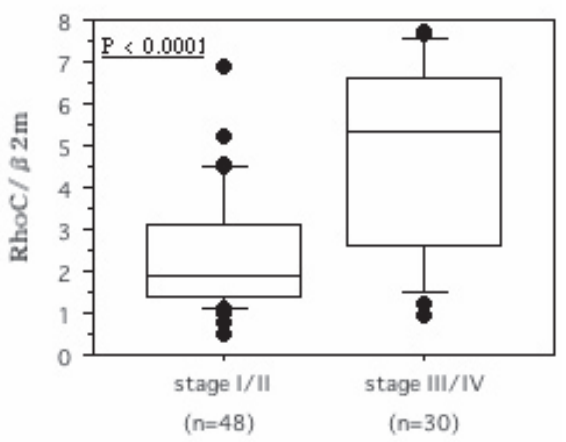

F. ROCK mRNA levels in Stage

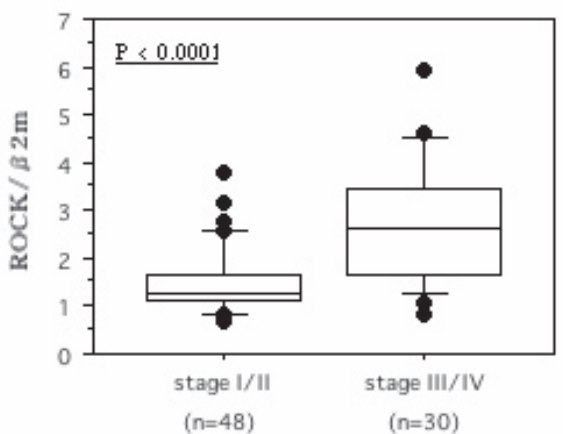

Fig. 2 Expression of RhoB, RhoC, and ROCK mRNAs in grade (A-C) and stage (D-F). The median value was seen in the box-plots.

survival. To our knowledge, this report is the first concerning the relationship between Rho and clinical important characteristics of RCC. The data suggested that the RhoC/ROCK pathway may be involved in progression of RCC.

Although our data showed a strong correlation of intensity of mRNA expression for RhoC with that for ROCK in tumors, this study was not designed to prove direct linkage between RhoC and ROCK expression. On the other hand, our findings demonstrated that mRNA expression for RhoC and ROCK was significantly higher in tumor tissues than in non-tumor tissues, while higher mRNA expression for either was linked with poor histological differentiation, higher stage, and poorer prognosis. Similarly, overexpression of RhoC has been associated with progression of pancreatic ductal adenocarcinoma (24) and inflammatory breast cancer (26). RhoC has been shown to be most associated with metastasis of cancer cells among Rho subfamily members according to studies using high-density DNA microarray (2). Furthermore, neovascularization is a characteristic feature in RCC, while the lung is the most common metastatic site for RCC. Overexpression of RhoC 
was reported to lead to increased expression of angiogenic factors (27), while RhoC overexpression also was found to promote the ability of melanoma cells to escape the circulation and colonize the lung (2). However, how RhoC induces metastasis remains unclear. While RhoA, RhoB, and RhoC share common functions in regulating the actin cytoskeleton

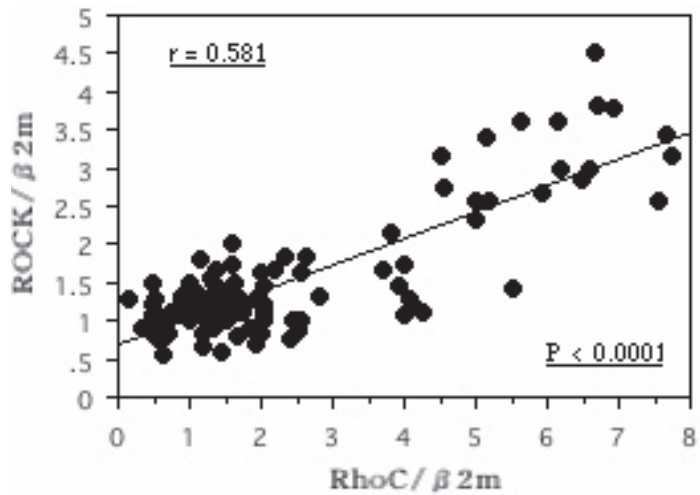

Fig. 3 Spearman rank correlation coefficient relationship between expression levels of mRNAs for RhoC and ROCK.
A : Overall survival in RhoB
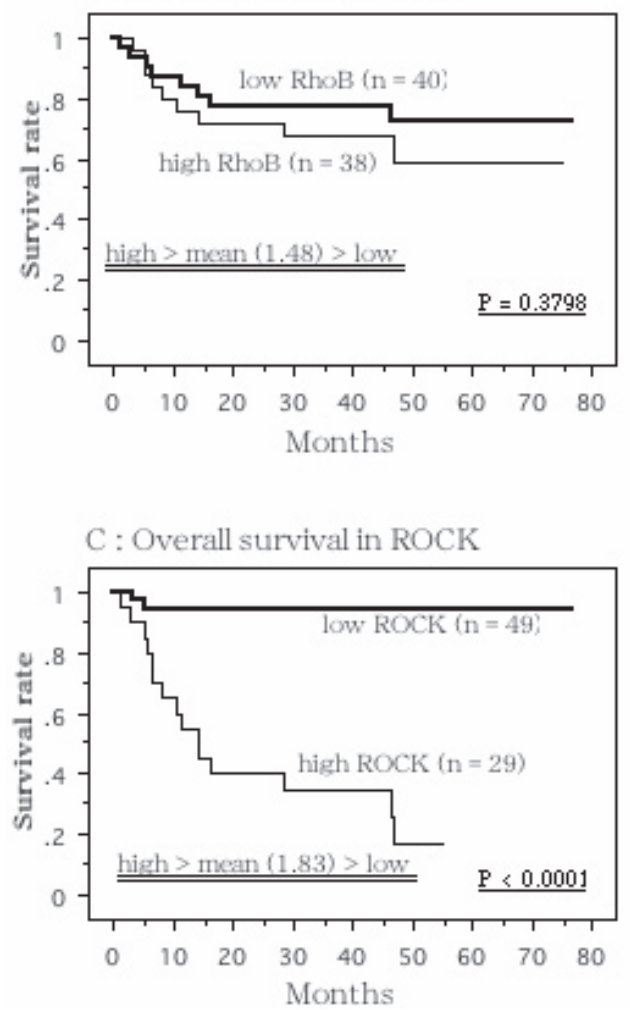

via the major downstream effector ROCK (22), they differ in subcellular location (1) and regulation of expression $(5,11)$. Therefore, tumor-specific and/or organ-specific roles for RhoA, RhoB, and RhoC may exist in human cancers.

The present observations associating higher expression of RhoC and ROCK mRNAs with advanced stage and poor survival are indicative of a possible role for the RhoC/ROCK pathway in renal carcinogenesis and progression of RCC. A specific ROCK inhibitor (Y-27632) was found to reduce invasive activity and dissemination of tumor cells (8, 10). Taking the various observations together, ROCK inhibitors may represent a potential therapy for prevention of cancer invasion and metastasis. Similarly to RhoC, mRNA expression for RhoB was higher in tumors than in non-tumor tissues; however, RhoB mRNA expression did not correlate with grade, stage, or prognosis. Recent studies indicate that RhoB actually might negatively regulate cell proliferation (4). A negative role in growth control and/or transformation would contrast with the positive effects of RhoA and RhoC in these processes

\section{B : Overall survival in RhoC}

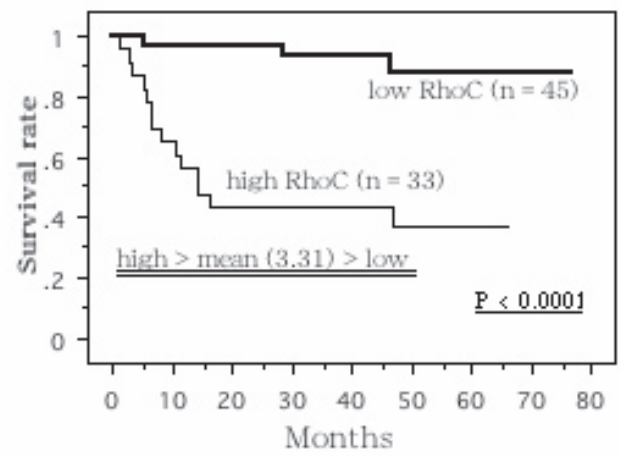

Fig. 4 Overall survival curve based on the mean values of mRNA expression for RhoB, RhoC, and ROCK in tumor tissues; the cases were divided into two groups at the mean values-high and low expression. $P$ value was analyzed by logrank test. 
Table 1 Cox regression analysis for potential prognostic factors in overall survival

\begin{tabular}{|c|c|c|c|c|c|c|}
\hline & Variable & $\begin{array}{l}\text { Unfavorable/ } \\
\text { favorable } \\
\text { characteristics }\end{array}$ & No. of Patients & Relative risk & $\begin{array}{c}95 \% \text { confidence } \\
\text { interval }\end{array}$ & $P$ value \\
\hline \multirow{4}{*}{$\begin{array}{l}\text { Univariate } \\
\text { analysis }\end{array}$} & Grade & $4,3 / 2 / 1$ & $17 / 36 / 25$ & 3.005 & $1.237-7.302$ & 0.0151 \\
\hline & $\mathrm{pT}^{*}$ & $4,3 / 2,1$ & $30 / 48$ & 7.185 & $2.052-25.157$ & 0.0021 \\
\hline & RhoC & high / low & $33 / 45$ & 4.272 & $1.116-9.596$ & 0.0048 \\
\hline & ROCK & high / low & $29 / 49$ & 4.327 & $1.780-11.940$ & 0.0023 \\
\hline \multirow{4}{*}{$\begin{array}{l}\text { Multivariate } \\
\text { analysis }\end{array}$} & Grade & $4,3 / 2 / 1$ & $17 / 36 / 25$ & 1.499 & $0.543-4.138$ & 0.435 \\
\hline & $\mathrm{pT}^{*}$ & $4,3 / 2,1$ & $30 / 48$ & 8.182 & $1.025-72.397$ & 0.0204 \\
\hline & RhoC & high / low & $33 / 45$ & 6.763 & $1.497-17.248$ & 0.0379 \\
\hline & ROCK & high / low & $29 / 49$ & 7.102 & $1.312-23.893$ & 0.0295 \\
\hline
\end{tabular}

$\mathrm{pT}^{*}$ : pathological Tumor classification of UICC TNM classification

(15). Recent advances indicate that RhoB is a specialized activator of apoptosis in transformed cells, and participates importantly in mediating cellular responses to farnesyltransferase inhibitors (FTIs) (21). Farnesyltransferase occupies a key position in the mevalonic acid pathway, resulting in conversion of mevalonic acid to Ras and Rho. Therefore, FTIs might prove useful in cancer therapy (21).

Although we presently could not analyze protein expression of Rho and ROCK, such determinations will be needed to more clearly elucidate the role of the RhoC/ROCK pathway and RhoB in a larger number of RCC. Such information may guide study of the effect of ROCK inhibitors and/or FTIs on RCC cells in vitro and in vivo.

\section{Acknowledgement}

The authors are grateful to Aki Yanagibayashi and Hitomi Yamazaki for their excellent technique in this study.

\section{REFERENCES}

1. Adamson P, Marshall CJ, Hall A and Tilbrook PA (1992) Post-translational modifications of $\mathrm{p} 21$ rho proteins. $J$ Biol Chem 267, 20033-20038.

2. Clark EA, Golub TR, Lander ES and Hynes RO (2000) Genomic analysis of metastasis reveals an essential role for RhoC. Nature 406, 532-535.

3. del Peso L, Hernandez-Alcoceba R, Embade N, Carnero A, Esteve P, Paje C and Lacal JC (1997) Rho proteins induce metastatic properties in vivo. Oncogene 15, 3047-3057.

4. Du W, Lebowitz P and Prendergast GC (1999) Cell growth inhibition by farnesyltransferase inhibitors is mediated by gain of geranylgeranylated RhoB. Mol Cell Biol 19, 18311840 .

5. Fritz G, Kaina B and Aktoriess K (1995) The ras-related small GTP-binding protein RhoB is immediate-early induc- ible by DNA damaging treatments. J Biol Chem 270, 25172 25177.

6. Fritz G, Just I and Kaina B (1999) Rho GTPase overexpressed in human tumors. Int J Cancer 81, 682-687.

7. Hall A (1998) Rho GTPases and the actin cytoskeleton. Science 279, 509-514.

8. Imamura F, Mukai M, Ayaki M and Akedo H (2000) Y-27632, an inhibitor of Rho-associated protein kinase, suppresses tumor cell invasion via regulation of focal adhesion and focal adhesion kinase. Jpn J Cancer Res 91, 811-816.

9. Ishizaki T, Naito M, Fujisawa K, Maekawa M, Watanabe N, Saito Y and Narumiya S (1997) p160ROCK, a Rho-associated coiled-coil forming protein kinase, works downstream of Rho and induces focal adhesions. FEBS Lett 404, 118-124.

10. Ito K, Yoshioka K, Akedo H, Uehata M, Ishizaki T and Narumiya $S$ (1999) An essential part for Rho-associated kinase in the transcellular invasion of tumor cells. Nat Med 5, 221225.

11. Jahner D and Hunter T (1991) The ras-related gene rhoB is an immediate-early gene inducible by v-Fps, epidermal growth factor, and platelet-derived growth factor in rat fibroblasts. Mol Cell Biol 1, 3682-3690.

12. Kamai T, Arai K, Sumi S, Tsujii T, Honda M, Yamanishi H and Yoshida K-I (2002) The rho/rho-kinase pathway is involved in the progression of testicular germ cell tumour. $B J U$ Int 89, 449-453.

13. Kamai T, Arai K, Tsujii T, Honda M and Yoshida K-I (2001) Overexpression of RhoA mRNA is associated with advanced stage in testicular germ cell tumor. BJU Int 87, 227-231.

14. Kamai T, Yanai Y, Arai K, Abe H, Yamanishi T, Kurimoto M and Yoshida K-I (2007) Increased interferon alpha receptor 2 mRNA levels is associated with renal cell carcinoma metastasis. BMC Cancer 2007, 7: 159.

15. Khosravi-Far R, Solski PA, Clark GJ, Kinch MS and Der CJ (1995) Activation of Rac1, RhoA, and mitogen-activated protein kinase are required for Ras transformation. Mol Cell Biol 15, 6443-6453.

16. Kimura K, Ito M, Amano M, Chihara K, Fukata Y, Nakafuku M, Yamamori B, Feng J, Nakano T, Okawa K, Iwamatsu A and Kaibuchi K (1996) Regulation of myosin phosphatase by Rho and Rho-associated kinase (Rho-kinase). Science 273, 245-248.

17. Motzer RJ, Bander NH and Nanus DM (1996) Renal-cell 
carcinoma. $N$ Engl J Med 335, 865-875.

18. Motzer RJ, Russo P, Nanus DM and Berg WJ (1997) Renal cell carcinoma. Current Problem Cancer 21, 189-232.

19. Nishimura J, Sakihara C, Zhou Y and Kanaide H (1996) Expression of rhoA and rho kinase mRNAs in porcine vascular smooth muscle. Biochem Biophys Res Com 227, 750-754.

20. Quigley JP and Armstrong PB (1998) Tumor cell intravasation alu-cidated; The chick embryo opens the window. Cell 94, 281-284.

21. Prendergast GC (2001) Actin'up: RhoB in cancer and apoptosis. Nat Rev Cancer 1, 162-168.

22. Ridley AJ and Hall A (1992) The small GTP-binding protein rho regulates the assembly of focal adhesions and actin stress fibers in response to growth factors. Cell 70, 389-399.

23. Sobin LH, editors (1997) International union against cancer. UICC, In: TNM Classification of Malignant Tumors, 5th ed., Wiley-Liss, New York.

24. Suwa H, Ohshio G, Imamura T, Watanabe G, Arii S, Imamura M, Narumiya S, Hiai H and Fukumoto M (1998) Overex- pression of the rho $\mathrm{C}$ gene correlates with progression of ductal adenocarcinoma of the pancreas. Br $J$ Cancer 77, 147-152.

25. Van Aelst L and D'Souza-Schorey C (1997) Rho GTPases and signaling networks. Genes Dev 11, 2295-2322.

26. van Golen KL, Wu Z-F, Qiao XT, Bao LW and Marajver SD (2000) RhoC GTPase, a novel transforming oncogene for human mammary epithelial cells that partially recapitulates the inflammatory breast cancer phenotype. Cancer Res 60,5832 5838 .

27. van Golen KL, Wu ZF, Qiao XT, Bo L and Merjver SD (2000) RhoC GTPase overexpression modulates induction of angiogenic factors in breast cells. Neoplasia 2, 418-422.

28. Yagoda A, Petrylak D and Thompson S (1993) Cytotoxic chemotherapy for advanced renal cell carcinoma. Urol Clin North Am 20, 303-321.

29. Yagoda A, Abi-Rached B and Petrylak D (1995) Chemotherapy for advanced renal-cell carcinoma: 1983-1993. Semin Oncol 22, 42-60. 
